\title{
Apresentando um instrumento de avaliação da comunicação à Fonoaudiologia Brasileira: Bateria MAC $* * * * * *$
}

\author{
Introducing a communication assessment tool to Brazilian speech \\ therapists: the MAC Battery
}

Rochele Paz Fonseca*

Maria Alice de Mattos Pimenta Parente**

Hélène Côté***

Bernadette Ska****

Yves Joanette*****

\begin{abstract}
*Fonoaudióloga e Psicóloga. Doutora em Psicologia do Desenvolvimento da Universidade Federal do Rio Grande do Sul (UFRGS / Universidade de Montreal). Professora Adjunto do Programa de Pós-Graduação em Psicologia - Cognição Humana da PUCRS. Endereço para correspondência: Av. Ipiranga, 6681 - Prédio 11 - Sala 938 - Porto Alegre - RS - CEP 90619-900 (rochele.fonseca@gmail.com).
\end{abstract}

**Fonoaudióloga. Doutora em Psicologia pela Universidade de São Paulo. Professora Adjunta do Programa de Pós-Graduação em Psicologia da UFRGS

***Fonoaudióloga e Psicóloga. Mestre em Fonoaudiologia - Universidade de Montreal. Pesquisadora da Faculté de Médecine, Université de Montréal Canadá.

****Fonoaudióloga. Ph.D em Psicologia - Universidade Católica de Louvain, Bélgica. Docente da Faculté de Médecine, Université de Montréal Canadá.

******Fonoaudiólogo. Ph.D em Ciências Neurológicas - Universidade de Montreal - Canadá. Diretor do Centre de Recherche du Institut Univesitaire de Gériatrie de Montréal, Faculté de Médecine, Université de Montréal - Canadá.

******Trabalho Realizado no Laboratório de Neuropsicolingüística, Instituto de Psicologia, Programa de Pós-Graduação em Psicologia (UFRGS), Brasil, e Centre de Recherche da Faculté de Médecine, Université de Montréal - Canadá.

Artigo de Revisão de Literatura e Revisão Sistemática

Artigo Submetido a Avaliação por Pares

Conflito de Interesse: não

Recebido em 11.10.2007.

Revisado em 11.03.2008; 06.07.2008.

Aceito para Publicação em 11.11.2008.

Referenciar este material como:

A . Fonseca RP, Parente MAMP, Côté H, Ska B, Joanette Y. Apresentando um instrumento de avaliação da comunicação à Fonoaudiologia Brasileira: Bateria MAC. PróFono Revista de Atualização Científica. 2008 out-dez;20(4):285-92

\begin{abstract}
Background: an assessment instrument to evaluate communication impairment after right brain damage: the Montreal Communication Evaluation Battery, an adapted brazilian version of the original canadian instrument - Protocole Montréal d'Évaluation de la Communication. Instruments that evaluate discursive, pragmatic, lexical-semantic and prosodic impairments are important for the diagnosis of communication disorders which are present in approximately $50 \%$ of the individuals with right brain damage. Systematic studies of the communication profile after lesions on this side of the brain have been carried out only during the last two decades. Aim: to present the Montreal Communication Evaluation Battery to brazilian speech therapists. Conclusion: the described instrument is an useful tool in the clinic for assessing four processes related to the communicative and linguistic abilities: discursive, pragmatic-inferential, lexicalsemantic and prosodic components. It is has been normalized, validated and its reliability has been confirmed. Although this instrument was developed and adapted for diagnosing communication disorders in individuals with right brain damage people, it can also be helpful in investigating communication sequels in traumatic brain injury, dementia, bilateral frontal lesions, left-brain damage, psychopathologies, such as schizophrenia, among others.
\end{abstract}

Key Words: Communication; Evaluation; Stroke; Neuropsychological Tests.

\section{Resumo}

Tema: um instrumento de avaliação de déficits comunicativos após lesão de hemisfério direito: Bateria Montreal de Avaliação da Comunicação, versão brasileira adaptada do instrumento original canadense Protocole Montréal d'Évaluation de la Communication. Ferramentas de avaliação dos déficits discursivos, pragmáticos, léxico-semânticos e prosódicos são necessárias para o diagnóstico dos distúrbios da comunicação presentes em aproximadamente $50 \%$ dos indivíduos lesados de hemisfério direito. O quadro comunicativo após acometimento desse lado do cérebro vem sendo estudado sistematicamente há apenas duas décadas. Objetivo: apresentar a Bateria Montreal de Avaliação da Comunicação aos fonoaudiólogos brasileiros. Conclusão: o instrumento apresentado mostra-se uma ferramenta clínica útil no exame das habilidades lingüísticas e comunicativas relacionadas a quatro processamentos: discursivo, pragmáticoinferencial, léxico-semântico e prosódico. Está normatizado, validado e sua fidedignidade foi confirmada. Embora tenha sido construído e adaptado para o exame dos distúrbios comunicativos em pacientes lesados de hemisfério direito, também pode auxiliar na investigação de seqüelas na comunicação em quadros de traumatismo crânio-encefálico, demência, lesões frontais bilaterais, lesões de hemisfério esquerdo, psicopatologias como a esquizofrenia, entre outros.

Palavras-Chave: Comunicação; Avaliação; Acidente Vascular Cerebral; Testes Neuropsicológicos. 


\section{Introdução}

O hemisfério cerebral esquerdo (HE) não é mais considerado exclusivo no processamento lingüístico. Essa exclusividade devia-se à noção de dominância cerebral, que propõe uma tendência hemisférica de controle do processamento da informação de uma função ${ }^{1}$. A importância da integridade do hemisfério direito (HD) para muitos componentes lingüísticos é atualmente reconhecida ${ }^{2}$.

A exclusividade do HE preponderou no estudo da linguagem até 1959, quando Eisenson sugeriu que uma lesão de HD poderia justificar uma seqüela comunicativa ${ }^{3}$. No entanto somente a partir do final dos anos 80 houve um aumento considerável das publicações sobre o papel do HD na linguagem ${ }^{3-5}$. Desde 1990 , década do cérebro ${ }^{6}$, as técnicas de neuroimagem sofreram um importante avanço ${ }^{7}$. Com esse aporte técnico-metodológico, muitas investigações foram realizadas com exames de neuroimagem sobre o processamento lingüístico, tanto em indivíduos calotomisados, hemisferectomizados e lesados cerebrais, como em neurologicamente saudáveis.

Tais estudos contribuíram muito para que os correlatos neurobiológicos da linguagem fossem revistos. De acordo com a noção inicial (século XIX), as regiões clássicas da linguagem são as Áreas de Broca, Wernicke e fascículo arqueado. Atualmente, referem-se áreas corticais associativas (regiões adjacentes às áreas clássicas da linguagem), estruturas subcorticais (tálamo, núcleos caudado, putâmen, giro do cíngulo), cerebelo e regiões do HD, algumas análogas às áreas de Broca e Wernicke ${ }^{8-9}$. Sabe-se, então, que há uma cooperação inter e intrahemisférica para uma comunicação efetiva.

Em suma, desde a década de 50 do século XX, além da associação clássica entre lesão de HE e afasia, correlatos de lesão de HD e déficits comunicativos têm sido cada vez mais freqüentes na literatura ${ }^{10-11}$. Mais sistematicamente há duas décadas, sintomas específicos de prejuízo em habilidades comunicativas vêm sendo relatados como parte da "síndrome" do $\mathrm{HD}^{12-13}$.

Os distúrbios de comunicação pós-lesão de HD podem envolver quatro processamentos comunicativos: discursivo, pragmático-inferencial, léxico-semântico e prosódico ${ }^{3}$, nos níveis compreensivo e expressivo. As alterações de produção discursiva incluem ausência de coerência, diminuição de conteúdo informativo e dificuldade com mudanças de temas. O discurso é tangencial e pouco claro. Quanto à compreensão discursiva, lesados de HD podem apresentar graves dificuldades em sintetizar ou inferir informações que não foram explicitadas ${ }^{14-15}$.

As alterações das habilidades pragmáticoinferenciais em lesados de HD talvez sejam as mais conhecidas ${ }^{16}$ : dificuldade em seguir regras conversacionais, como troca de turnos comunicativos e compartilhamento de informações, em considerar adequadamente pistas contextuais para a compreensão de emissões não-literais, tais como atos de fala indiretos, metáforas, humor ou sarcasmo ${ }^{10}$.

Quanto aos distúrbios do processamento léxicosemântico, a compreensão e a produção de palavras podem estar alteradas, principalmente quanto menor for sua frequiência e concretude ${ }^{4}$. Uma lesão de HD também pode ocasionar uma dificuldade em compreender palavras metafóricas ${ }^{17} \mathrm{e}$ em identificar adequadamente relações funcionais ou categoriais entre palavras ${ }^{18}$.

Por fim, os distúrbios do processamento prosódico englobam déficits na compreensão e na produção de entonações emocionais e linguíísticas ${ }^{11,19}$. Os lesados de HD podem apresentar uma fala com entonação diminuída ou ausente ou não distinguir adequadamente entonações lingüísticas (por exemplo, interrogação de ordem) e emocionais (por exemplo, alegria de surpresa).

Aproximadamente $50 \%$ dos lesados de HD apresenta distúrbios comunicativos adquiridos ${ }^{20-}$ ${ }^{21}$, resultando em uma desvantagem comunicativa significativa. A perda da integridade do HD devido a uma lesão cérebro-vascular e os conseqüentes distúrbios em vários componentes comunicativos afetam as interações sociais e geram um importante impacto psicossocial e funcional ${ }^{3,22}$. Estes indivíduos formam, portanto, uma importante população neurológica que deve ser definida para ser encaminhada a centros de reabilitação.

Existem controvérsias na literatura quanto à exclusividade do HD nos déficits comunicativos acima descritos. Alguns autores relatam distúrbios de compreensão de linguagem não-literal em afásicos, tanto na interpretação de expressões idiomáticas ${ }^{23}$ como na de atos de fala indiretos ${ }^{24}$. Duas hipóteses podem ser levantadas:

1. Lesados de HE podem desempenhar com menor êxito tarefas de linguagem figurada por não processarem os componentes lingüísticos formais, como os aspectos sintáticos.

2. A comunicação bem-sucedida se dá graças a uma cooperação inter-hemisférica; portanto, o processamento não-literal da linguagem também deve depender da ativação de áreas do HE. 
Essa segunda hipótese é corroborada pelos achados de um estudo com ressonância magnética funcional sobre reconhecimento prosódico emocional $^{25}$. Foram encontrados três estágios de ativação, sendo os dois primeiros no HD e o terceiro no HE. Em pesquisas em que indivíduoscontrole, lesados de HD e lesados de HE são avaliados, observa-se uma tendência dos lesados de HE terem um desempenho inferior aos sujeitoscontrole, mas superior aos lesados de $\mathrm{HD}^{26}$. Assim, é necessário, ainda, um melhor entendimento do perfil comunicativo de lesados de HD, das suas possíveis semelhanças com lesados de $\mathrm{HE}$ e da cooperação inter-hemisférica na comunicação.

O único consenso na literatura é que as alterações comunicativas pós-lesão de HD não podem ser consideradas déficits afásicos clássicos. Os lesados de HD apresentam os aspectos fonológicos, morfológicos, sintáticos e semânticos literais preservados.

De tal modo, os testes tradicionalmente elaborados para a avaliação da linguagem em quadros de afasia não apresentam tarefas nem estímulos sensíveis o suficiente para detectarem as alterações comunicativas pós-lesão de HD. Entretanto, antes da década de 1980, os clínicos avaliavam seus pacientes lesados de HD com instrumentos de avaliação da afasia, pois não havia testes específicos padronizados para o exame das funções cognitivas e/ou comunicativas de especialização desse hemisfério até 1985. A ausência de instrumentos provavelmente contribuiu para a demora em se estudar os distúrbios comunicativos pós-lesão de HD.

De 1985 até o final da década de 1990, alguns instrumentos foram construídos para a avaliação de habilidades cognitivas e/ou comunicativas relacionadas ao HD: RICE (Rehabilitation Institute of Chicago Evaluation of Communication Problems in Right Hemisphere Dysfunction), em 1985; RIPA (Ross Information Processing Assessment), em 1986; Pragmatic Protocol, em 1987; MIRBI (Mini Inventory of Right Brain Injury), em 1989; e, RHLB (Right Hemisphere Language Battery), em 1989. Essas baterias avaliam percepção visual, percepção e esquema corporais, processamento visuo-espacial, memória de curto prazo, orientação têmporo-espacial, discurso narrativo, compreensão metafórica, entre outras habilidades neuropsicológicas gerais. A maioria, com exceção da RHLB, inclui poucas tarefas que examinam o processamento lingüístico realizado especificamente pelo HD, apresentando limitações teóricas e/ou metodológicas. As primeiras são representadas principalmente pela base teórica desatualizada: como todas as baterias basearam-se na literatura da década de 1980 ou de antes, sua construção não foi norteada por avanços teóricos importantes nas áreas de psicologia cognitiva e psicolingüística. As limitações metodológicas podem ser exemplificadas pela inclusão de poucas tarefas ou poucos estímulos por dimensão comunicativa e pela presença predominante de tarefas visuo-espaciais. Um comentário geral ainda pode ser feito: os instrumentos mencionados estão publicados na língua inglesa, não havendo testes originalmente editados em línguas latinas, o que exige maior cuidado em sua adaptação ${ }^{3}$.

Deste modo, observa-se que mesmo após a importante evolução da avaliação das funções do HD, permanece uma demanda clínica: quanto à qualidade de avaliações para os distúrbios de linguagem pós-lesões de HD e à necessidade de adaptações para diferentes línguas. Essa demanda pode justificar, pelo menos em parte, o fato da atuação clínica com lesados de HD ainda consistir em uma prática fonoaudiológica pouco desenvolvida ${ }^{22}$. Neste contexto, um grupo canadense construiu o Protocole d'Évaluation de la Communication, Protocole MEC, publicado em francês em 2004,22.

No Brasil, até onde se tem conhecimento, não há instrumentos disponíveis para a avaliação das habilidades comunicativas que podem estar afetadas em quadros de lesão de HD. A afasia é um dos distúrbios neurológicos adquiridos de linguagem mais estudados. Não surpreende, então, o maior enfoque dado aos testes de avaliação das habilidades lingüísticas de especialidade do HE na literatura nacional e sua conseqüente maior utilização na clínica ${ }^{27-29}$. Em busca da redução desta demanda, o presente artigo visa a apresentar a Bateria Montreal de Avaliação da Comunicação Bateria MAC, versão do Protocole MEC adaptado ao Português Brasileiro ${ }^{30}$, relevante pelas seguintes justificativas:

. esse é o primeiro instrumento de avaliação comunicativa relacionada ao HD adaptado para uso no Brasil;

. essa bateria foi lançada pela Editora Pró-Fono em 2008, sendo bastante recente;

. descrições clínicas dessa população neurológica ainda são escassas, devendo a fonoaudiologia brasileira se apropriar mais da avaliação em quadros de alterações comunicativas pós-lesão de HD;

. o aumento das descrições clínicas dos distúrbios comunicativos em lesados de HD levará a uma 
sensibilização e conscientização dos profissionais da área da saúde quanto à necessidade de encaminhamento desses pacientes à reabilitação; - programas de reabilitação específicos poderão ser formulados com uma ferramenta clínica padronizada que complemente o exame comunicativo nesse quadro.

Bateria Montreal de Avaliação da Comunicação Bateria MAC

O Protocole MEC $^{3,22}$ foi elaborado com o objetivo de avaliar quatro processamentos comunicativos: discursivo; pragmático-inferencial; léxico-semântico; prosódico.

É composto por 14 tarefas: questionário sobre a consciência das dificuldades; discurso conversacional; interpretação de metáforas; evocação lexical livre; prosódia lingüística compreensão; prosódia lingüística repetição; discurso narrativo; evocação lexical com critério ortográfico; prosódia emocional compreensão; prosódia emocional repetição; interpretação de atos de fala indiretos; evocação lexical com critério semântico; prosódia emocional produção; julgamento semântico. Na Figura 1, observa-se a distribuição das tarefas conforme os processamentos que avaliam. O objetivo de cada subteste é descrito no Quadro 1.

O Protocole MEC foi padronizado com 180 indivíduos neurologicamente saudáveis de diferentes idades (39 a 85 anos) e níveis educacionais ( 0 a 30 anos de estudo formal). Mostrou boa fidedignidade pela precisão entre avaliadores e adequada validade de conteúdo ${ }^{22}$. Foi ou está sendo adaptado para uso nos países: Argentina, França, Irã, Itália, Suíça e Estados Unidos da América, etc.

A versão brasileira, Bateria MAC, foi normatizada com 300 indivíduos não-lesados, de 19 a 75 anos de idade, com 2 a 35 anos de escolaridade. Sua adequada confiabilidade foi confirmada, com evidências de validades de conteúdo, construto e critério ${ }^{30}$.
Em seu manual de aplicação e pontuação, há normas detalhadas de como aplicar, registrar e interpretar cada tarefa. Estabeleceu-se um ponto de alerta para cada grupo normativo: três grupos etários (19-39 anos, 40-59 anos e 60-75 anos), subdivididos em dois grupos de escolaridade (2-7 anos de estudo e 8 anos ou mais). Com base nesse ponto de alerta, escore a partir do qual o examinador deve supor que os déficits comunicativos encontrados estão relacionados à lesão de $\mathrm{HD}^{22}$, o clínico poderá diagnosticar alterações comunicativas. Tal diagnóstico deve, obviamente, ser complementado por uma anamnese bem detalhada, por um instrumento de avaliação da comunicação funcional, que verifique o impacto do déficit comunicativo no dia-a-dia e o grau de independência do paciente, e pela observação e impressão clínica. As tarefas da Bateria MAC encontram-se descritas brevemente na Quadro 2.

Além das normas de interpretação quantitativa para cada subteste, no manual de aplicação e pontuação, há, também, algumas sugestões de análise qualitativa. Por exemplo, na tarefa Evocação lexical livre, sugere-se que sejam examinadas a presença de erros, como repetição de palavras, o que poderia indicar perseveração, e a estratégia de exploração utilizada, tais como ortográfica ou semântica.

A Bateria MAC vem acompanhada por uma triagem dos distúrbios comunicativos que complementa o exame da comunicação de populações neurológicas. Essa triagem é composta por uma questão aberta e 15 questões de sim e não que investigam as modificações do padrão comunicativo do indivíduo, pela consulta a um familiar, amigo e/ou cuidador que faça uma comparação da comunicação do paciente pré e pós-lesão.

FIGURA 1. Diagrama da distribuição das 14 tarefas da Bateria MAC por processamento comunicativo.

\begin{tabular}{|c|c|c|c|}
\hline \multicolumn{4}{|c|}{ Processamento Comunicativo } \\
\hline processamento discursivo & $\begin{array}{c}\text { processamento pragmático- } \\
\text { inferencial }\end{array}$ & processamento léxico-semântico & processamento prosódico \\
\hline $\begin{array}{l}\text { discurso conversacional; } \\
\text {. discurso narrativo. }\end{array}$ & $\begin{array}{l}\text { interpretação de metáforas; } \\
\text { interpretação de atos de fala } \\
\text { indiretos; } \\
\text {. discurso conversacional. }\end{array}$ & $\begin{array}{c}\text {. evocação lexical; } \\
\text {.julgamento semântico; } \\
\text { interpretação de metáforas. }\end{array}$ & $\begin{array}{l}\text { prosódia lingüística; } \\
\text { prosódia emocional. }\end{array}$ \\
\hline
\end{tabular}


QUADRO 1. Objetivos dos subtestes da Bateria MAC.

\begin{tabular}{|l|l|}
\hline \multicolumn{1}{|c|}{ Tarefas } & \multicolumn{1}{c|}{ Objetivos } \\
\hline $\begin{array}{l}\text { questionário sobre a consciência das } \\
\text { dificuldades }\end{array}$ & $\begin{array}{l}\text { investigar a consciência dos distúrbios de linguagem e o seu impacto na vida cotidiana dos } \\
\text { indivíduos com lesão neurológica, ou seja, a presença de anosognosia (quadro em que o paciente } \\
\text { não reconhece ou reconhece apenas parcialmente suas seqüelas). } \\
\text { discurso conversacional }\end{array}$ \\
examinar o comportamento verbal e não-verbal em situação de conversação. \\
interpretação de metáforas & verificar a compreensão da linguagem não-literal em sentenças. \\
evocação lexical & avaliar a produção de vocábulos livre, ortográfica e semanticamente. \\
prosódia lingüística & investigar a compreensão e a produção repetida de afirmação, interrogação e ordem. \\
prosódia emocional & avaliar a compreensão e a produção repetida e espontânea das emoções raiva, alegria e tristeza. \\
discurso narrativo & examinar o reconto e a compreensão de uma narrativa. \\
interpretação de atos de fala indiretos & averiguar a compreensão não-literal da intenção de interlocutores em situações comunicativas. \\
julgamento semântico & analisar a habilidade de identificar e explicar relações semânticas entre duas palavras. \\
\hline
\end{tabular}

QUADRO 2. Descrição das 14 tarefas da Bateria MAC.

\begin{tabular}{|c|c|c|}
\hline $\begin{array}{l}\text { Processamento } \\
\text { Comunicativo }\end{array}$ & Tarefa & Descrição \\
\hline \multirow[b]{2}{*}{ prosódico } & $\begin{array}{ll} & \text { compreensão }(/ 12) \\
\text { prosódia } & \\
\text { lingüística } & \text { repetição }(/ 12)\end{array}$ & $\begin{array}{l}12 \text { sentenças pré-registradas em áudio. O paciente identifica a } \\
\text { entonação com apoio em três Figuras indicando: afirmação, } \\
\text { pergunta e ordem. } \\
\text { Mesmos estímulos da anterior. Repetem-se as sentenças. }\end{array}$ \\
\hline & $\begin{array}{l}\text { repetição }(/ 12) \\
\text { produção }(/ 18)\end{array}$ & $\begin{array}{l}12 \text { sentenças pré-registradas em áudio. O paciente identifica a } \\
\text { entonação com apoio em três faces indicando: tristeza, alegria e } \\
\text { raiva. } \\
\text { Mesmos estímulos da anterior. Repetem-se as sentenças. } \\
9 \text { curtos textos com situações comunicativas induzindo a uma } \\
\text { emoção ( } 3 \text { situações - alegria, tristeza e raiva, com } 3 \text { sentenças- } \\
\text { alvo). O paciente produz oralmente a sentença-alvo com a } \\
\text { entonação apropriada. }\end{array}$ \\
\hline \multirow[t]{2}{*}{ léxico-semântico } & $\begin{array}{l}\text { livre } \\
\text { com critério ortográfico } \\
\text { com critério semântico }\end{array}$ & $\begin{array}{l}\text { O paciente diz o máximo possível de palavras em dois minutos e } \\
\text { meio. } \\
\text { O paciente diz o máximo possível de palavras que começam com a } \\
\text { letra "p" em dois minutos. } \\
\text { O paciente diz o máximo possível de vocábulos que sejam roupas } \\
\text { em dois minutos. }\end{array}$ \\
\hline & julgamento semântico (/24) & $\begin{array}{l}24 \text { pares de palavras, } 12 \text { com e } 12 \text { sem relação semântica categorial. } \\
\text { o paciente indica se há ou não relação semântica e, caso haja, } \\
\text { explica qual é essa relação. }\end{array}$ \\
\hline \multirow{2}{*}{ discursivo } & discurso conversacional (/34) & $\begin{array}{l}\text { conversação por } 10 \text { minutos entre paciente e examinador sobre dois } \\
\text { tópicos diferentes; análise sistemática de } 17 \text { variáveis } \\
\text { comunicativas. }\end{array}$ \\
\hline & discurso narrativo (/13) & $\begin{array}{l}\text { narrativa com cinco parágrafos, recontada parágrafo por parágrafo e } \\
\text { de modo integral, com } 12 \text { questões de compreensão, solicitação de } \\
\text { título e análise de processamento de inferências. }\end{array}$ \\
\hline \multirow[t]{2}{*}{ pragmático } & interpretação de metáforas (/40) & $\begin{array}{l}20 \text { metáforas: } 10 \text { metáforas novas (não lexicalizadas) e } 10 \\
\text { expressões idiomáticas (lexicalizadas). o paciente explica cada } \\
\text { sentença e responde a uma questão de múltipla escolha (alternativas } \\
\text { de explicação). }\end{array}$ \\
\hline & interpretação de atos de fala indiretos (/40) & $\begin{array}{l}20 \text { situações: } 10 \text { com um ato de fala direto e } 10 \text { com um indireto. o } \\
\text { paciente explica a intenção do interlocutor e responde a uma questão } \\
\text { de múltipla escolha (alternativas de explicação). }\end{array}$ \\
\hline $\begin{array}{l}\text { consciência das } \\
\text { dificuldades }\end{array}$ & $\begin{array}{l}\text { questionário sobre a consciência das } \\
\text { dificuldades (/7) }\end{array}$ & $\begin{array}{l}7 \text { questões do tipo sim-não sobre a consciência do paciente das suas } \\
\text { possíveis seqüelas. }\end{array}$ \\
\hline
\end{tabular}


Essa ferramenta clínica está disponibilizada pela Editora Pró-Fono aos fonoaudiólogos e neuropsicólogos brasileiros em 2008. Seu material inclui manual introdutório (contexto teórico e dados psicométricos), manual de aplicação e de pontuação, protocolo de registro, livro de estímulos e cd-rom com estímulos prosódicos e escritos. Para o exame com a triagem dos distúrbios comunicativos, acompanham duas versões: uma preenchida pelo clínico e outra diretamente pelo próprio familiar, amigo ou cuidador.

Por fim, explicita-se porque o Protocole MEC foi escolhido para ser adaptado ao Português Brasileiro dentre as outras ferramentas internacionais:

. seu aporte teórico inclui os avanços significativos nas áreas de psicologia cognitiva, psicolingüística e técnicas de neuroimagem que ocorreram desde a década de 1990;

. o Protocole MEC avalia os quatro processamentos comunicativos que podem estar afetados pós-lesão de HD, diferentemente da maioria das demais baterias, que priorizam um ou outro componente; .é o único publicado em uma língua de origem latina, facilitando sua adaptação para outras línguas de mesma origem;

. a seleção das tarefas do Protocole MEC baseouse no estudo das manifestações clínicas da população de lesados de HD;

. o protocolo é relativamente fácil de ser aplicado e pontuado, do tipo lápis e papel, com duração média de duas sessões de 45 minutos;

- as normas de pontuação foram feitas para cada tarefa, permitindo ao clínico a aplicação isolada de alguns subtestes.

Apesar da rigorosa qualidade clínica e científica do Protocole MEC, seus próprios autores mencionam duas limitações ${ }^{3}$. Não oferece uma avaliação exaustiva dos componentes comunicativos. A compreensão e produção de humor e sarcasmo, por exemplo, é apenas indiretamente avaliada no discurso conversacional, sem ser foco de um subteste específico. Além disso, como não tem por objetivo avaliar outros déficits cognitivos que podem caracterizar o quadro clínico pós-lesão de HD, como um déficit de atenção, percepção visuo-espacial ou de memória de trabalho, não especifica a causa subjacente ao distúrbio de linguagem. Por isso, deve ser complementado com uma avaliação neuropsicológica mais ampla.

\section{Conclusão}

A Bateria MAC mostra-se uma ferramenta clínica válida e confiável no processo de avaliação de habilidades comunicativas discursivas, pragmáticas, léxico-semânticas e prosódicas. O uso de uma bateria de comunicação validada, juntamente com tarefas neuropsicológicas, possibilitará descrever diferentes alterações comunicativas que podem ocorrer após acidentes vasculares cerebrais no HD, assim como em outros quadros neurológicos.

Com o uso da Bateria MAC no Brasil, os seguintes objetivos poderão ser alcançados em próximos estudos:

. contribuir para a descrição de diferentes tipos de alterações comunicativas pós-lesão de HD;

. identificar os locais de lesão associados;

. relacionar os perfis de distúrbios de comunicação a alterações cognitivas subjacentes;

. verificar as reais dificuldades de adaptação na vida diária de cada subgrupo clínico;

. fornecer subsídios para o planejamento de estratégias adequadas na reabilitação e adaptação do paciente;

- averiguar a eficácia de programas de reabilitação, entre outros.

A Bateria MAC foi adaptada para o exame dos distúrbios comunicativos em pacientes lesados de HD, podendo auxiliar na avaliação da comunicação em quadros de traumatismo crânio-encefálico, demência, lesões frontais bilaterais, lesões unilaterais de HE, tumores cerebrais, lesões cerebelares, psicopatologias como a esquizofrenia e a Síndrome de Asperger, entre outros.

Ressalta-se que a prática de construção de instrumentos fonoaudiológicos brasileiros e de adaptação de ferramentas clínicas internacionais para o Português Brasileiro é ainda incipiente. Para que a área de avaliação da linguagem e da comunicação cresça ainda mais, o uso de testes precisos, válidos e sensíveis é extremamente importante para complementar um processo diagnóstico acurado. 
Agradecimentos: ao Fomento Conselho Nacional de Desenvolvimento Científico e Tecnológico (CNPq) e Coordenação de Aperfeiçoamento de Pessoal de Nível Superior (CAPES).

\section{Referências Bibliográficas}

1. Powell HW, Parker GJ, Alexander DC, Symms MR, Boulby PA, Wheeler-Kingshott CA, et al. Abnormalities of language networks in temporal lobe epilepsy. Neuroimage. 2007;36(1):209-21.

2. Myers PS. Profiles of communication deficits in patients with right cerebral hemisphere damage: implications for diagnosis and treatment. Aphasiology. 2005;19:1147-60.

3. Côté H, Payer M, Giroux F, Joanette Y. Towards a description of clinical communication impairment profiles following right-hemisphere damage. Aphasiology. 2007;21(6,7,8):739-49.

4. Rainville P, Goulet P, Joanette Y. Contribution of the right hemisphere to the processing of concrete words. Clin Aphasiol. 1995;23:207-16.

5. Van Lancker D. Rags to riches: our increasing appreciation of cognitive and communicative abilities of the human right cerebral hemisphere. Brain Lang. 1997;57:1-11.

6. Baddeley A, Bueno O, Cahill L, Fuster JM, Izquierdo I, Mcgaugh JL, et al. The brain decade in debate: I. Neurobiology of learning and memory. Braz J Med Biol Res. 2000;33:993-1002.

7. Gernsbacher MA, Kaschak MP. Neuroimaging studies of language production and comprehension. Ann Rev Psychol. 2003;54:91-114.

8. Stowe LA, Haverkof M, Zwarts F. Rethinking the neurological basis of language. Lingua. 2005;115(7):9971042 .

9. Booth JR, Wood L, Lu D, Houk JC, Bitan T. The role of the basal ganglia and cerebellum in language processing. Brain Res. 2007;1133(1):136-44.

10. Zaidel E, Kasher A, Soroker N, Batori G. Effects of right and left hemisphere damage on performance of the "Right Hemisphere Communication Battery". Brain Lang. 2002;80:510-35

11. Pell MD. Reduced sensitivity to prosodic attitudes in adults with focal right hemisphere brain damage. Brain Lang. 2007;101(1):64-79.

12. Blake ML, Duffy JR, Tompkins CA, Myers PS. Right hemisphere syndrome is in the eye of the beholder. Aphasiology. 2003;17(5):423-32.

13. Myers PS. Toward a definition of RHD syndrome. Aphasiology. 2001;15(10/11):913-8.

14. Marini A, Carlomagno S, Caltagirone C, Nocentini U. The role played by the right hemisphere in the organization of complex textual structures. Brain Lang. 2005;93:4654.

15. Brady M, Armstrong L, Mackenzie C. An examination over time of language and discourse production abilities following right hemisphere brain damage. J Neurolinguist. 2006;19(4):291-310.
16. Champagne M, Desautels M, Joanette Y. Accounting for the pragmatic deficit in RHD individuals: a multiple case study. Brain Lang. 2003;87:210-1

17. Gagnon L, Goulet P, Giroux F, Joanette Y. Processing of metaphoric and non-metaphoric alternative meanings of words after right- and left-hemispheric lesion. Brain Lang. 2003;87:217-26.

18. Nocentini U, Goulet P, Roberts PM, Joanette Y. The effects of left- versus right-hemisphere lesions on the sensitivity to intra- and interconceptual semantic relationships. Neuropsychol. 2001;39:443-51.

19. Ross ED, Monnot M. Neurology of affective prosody and its functional-anatomic organization in right hemisphere. Brain Lang. 2008;104(1):51-74.

20. Joanette Y, Goulet P, Daoust H. Incidence et profils des troubles de la communication verbale chez les cérébrolésés droits. Rev Neuropsychol. 1991;1(1):3-27.

21. Benton E, Bryan K. Right cerebral hemisphere damage: incidence of language problems. Int Jf Rehab Res. 1996;19:47-54.

22. Côté H, Moix V, Giroux F. Évaluation des troubles de la communication des cérébrolésés droits. Rééduc Orthoph. 2004;219(4):107-22.

23. Papagno C, Caporali A. Testing idiom comprehension in aphasic patients: the effects of task and idiom type. Brain Lang. 2007;100:208-20.

24. Soroker N, Kasher A, Giora R, Batori G, Corn C, Gil M, et al. Processing of basic speech acts following localized brain damage: a new light on the neuroanatomy of language. Brain Cogn. 2005;57(2):214-7.

25. Wildgruber D, Ackermann H, Kreifelts BE, Ethofer T. Cerebral processing of linguistic and emotional prosody: fMRI studies. Prog Brain Res. 2006;156:249-68.

26. Cutica H, Bucciarelli M, Bara BG. Neuropragmatics: extralinguistic pragmatic ability is better preserved in lefthemisphere-damaged patients than in right-hemispheredamaged patients. Brain Lang. 2006;98:12-25.

27. Radanovic M, Mansur LL, Azambuja MJ, Porto CS, Scaff M. Contribution to the evaluation of language disturbances in subcortical lesions: a piloty study. Arq NeuroPsiquiatr. 2004;62(1):51-7.

28. Mansur LL, Radanovic M, Araujo GC, Taquemori LY, Greco LL. Teste de nomeação de Boston: desempenho de uma população de São Paulo. Pró-Fono R Atual Cient. 2006;18(1):13-20.

29. Santos AD, Ortiz KZ. Comparação do desempenho de pacientes afásicos em diferentes testes de compreensão oral. Fono Atual. 2005;8(33):27-33.

30. Fonseca RP, Parente MAMP, Côté H, Joanette Y. Processo de adaptação da Bateria Montreal de Avaliação da Comunicação - Bateria MAC - ao Português Brasileiro. Psicol Refl Crít. 20(2). In press 2008. 